\title{
EL NUEVO TEXTO REFUNDIDO DE LA LEY GENERAL DE LA SEGURIDAD SOCIAL... O LA HISTORIA INTERMINABLE
}

\author{
Faustino Cavas MarTínez \\ Departamento de Derecho del Trabajo \\ y de la Seguridad Social \\ Facultad de Derecho de la Universidad de Murcia \\ cavas@um.es
}

\section{CONSIDERACIONES PREVIAS}

En el BOE del pasado 31 de octubre de 2015 apareció publicado el Real Decreto Legislativo 8/2015, de 30 de octubre, mediante el que se aprueba un nuevo texto refundido de la Ley General de la Seguridad Social (TRLGSS/2015).

Se trata de la tercera refundición que a lo largo de su ya dilatada historia ha conocido el principal exponente de la producción normativa que regula el sistema español de protección social (la Ley General de Seguridad Social); una norma, por otra parte, largamente esperada, pues hacía tiempo que se venía reclamando la necesidad y urgencia de aprobar un nuevo corpus iuris que integrase de forma armonizada y sistemática el confuso piélago de disposiciones legales que habían ido apareciendo desde la entrada en vigor del texto refundido de la LGSS aprobado por Real Decreto Legislativo 1/1994, de 20 de junio -TRLGSS/1994—, en unos casos adoptando reformas que accedían directamente al texto del mencionado texto refundido, distorsionando su estructura a base de incrustar preceptos de numeración poco comprensible y una lista interminable de disposiciones adicionales, mientras que en otros muchos casos, y esto era lo más preocupante desde el punto de vista de la seguridad jurídica, las nuevas previsiones quedaban extramuros del TRLGSS/1994, ubicadas en disposiciones de la más variada laya, obligando de este modo a los operadores jurídicos a desarrollar una penosísima labor de rastreo y localización del grupo normativo y/o del precepto aplicable en un momento determinado a una situación concreta, con grave perjuicio de los asegurados o beneficiarios en caso de error. 
La entrada en vigor del TRLGSS/2015 tuvo lugar el 2 de enero de 2016, quedando derogado a partir de ese día el TRLGSS/1994, que acumuló una vigencia de algo más de veinte años.

\section{ANTECEDENTES}

El Derecho de la Seguridad Social constituye una disciplina de relevancia indiscutible, integrada por instituciones de gran complejidad, que se presenta con tintes esotéricos para quien no es especialista; quien se aventura en sus senderos se ve absorbido de inmediato por una colosal normativa de dispar rango y cronología. Además de extenso, el ordenamiento de la Seguridad Social es complejo, y ello no tanto —o no sólo- por la dificultad de los conceptos que se manejan en esta rama del Derecho, cuanto por el muy elevado número de piezas que integran la maquinaria de sus instituciones (Martín Valverde, 1999, 361).

La ordenación histórica del sistema de Seguridad Social se ha realizado atendiendo a criterios de agrupación (cuando no superposición) de normas, que han ido apareciendo y variando a lo largo del tiempo. A ello ha contribuido considerablemente la técnica de legislación en casca$d a$, que, asociada al fenómeno de legislación delegada, se ha venido practicando desde comienzos de los años sesenta: el legislador delegante remitía al gobierno la elaboración de la legislación delegada (ley de bases/texto articulado), evidenciando con ello su incapacidad para asumir la regulación de una materia tan compleja como la Seguridad Social, pero, a su vez, la legislación delegada encomendaba su desarrollo a los reglamentos generales que debían ser aprobados por decreto y que, a su vez, preveían que el ministro de Trabajo dictaría las correspondientes órdenes de aplicación, las cuales, por su parte, autorizaban a los órganos administrativos inferiores para resolver las cuestiones que pudieran plantearse en su aplicación, abriendo así la vía para el «derecho subterráneo» de la Seguridad Social (Desdentado Bonete, 1996, 478). De este modo, la causa que más influye en la dificultad de conocimiento de la Seguridad Social es, al igual que ocurre con el Derecho del Trabajo, la movilidad casi vertiginosa de este sector normativo. Las reformas se suceden sin cesar a partir de las exigencias y de las presiones del momento, y con un encuadramiento en muchos casos insólito dentro o fuera de lo que podríamos considerar como el marco normal del Derecho de la Seguridad Social («deslocalización normativa»). 
Cabe recordar que la originaria Ley General de la Seguridad Social procede del texto articulado I, como resultado de la delegación otorgada al Gobierno por la Ley 193/1963, de 28 de diciembre, de Bases de la Seguridad Social, y que terminó siendo aprobado mediante Decreto 907/1966, de 21 de abril. Fue la LSS-1966 la que implantó en España un verdadero Sistema de Seguridad Social, superando las limitaciones del precedente régimen de seguros sociales; un régimen al que se venía reprochando, con razón, la insuficiencia de su ámbito de aplicación personal, el tratamiento fraccionado y no conjunto que deparaba a los riesgos cubiertos, las múltiples y también fraccionadas fuentes de financiación, y la diversidad de entidades y cajas gestoras (Montoya Melgar, 2016, 17). Años más tarde, la Ley 24/1972, de 21 de junio, de financiación y perfeccionamiento de la acción protectora del Régimen General de la Seguridad Social, introdujo modificaciones en la ordenación jurídica inicial, incidiendo tanto sobre las contingencias y situaciones protegidas como en el ámbito específico de la financiación del sistema, extendiéndose no sólo al Régimen General, sino también sobre los sistemas especiales (Ramos, 2016, 1). Bajo estas circunstancias, la propia Ley 24/1972 contempló la necesidad de llevar a cabo una refundición de la LSS de 1966 con la de 1972 y los preceptos en materia de Seguridad Social que figurasen en otras disposiciones de igual rango, dando lugar al primer texto refundido de la Ley General de la Seguridad Social, aprobado por Decreto 2061/1974, de 30 de mayo (TRLGSS/1974).

Pero la promulgación del TRLGSS/1974, que se mantuvo en vigor durante veinte años, no supuso un descenso en la producción normativa en materia de Seguridad Social, que siguió imparable, especialmente tras la aprobación de la Constitución Española en 1978, de un lado, para desarrollar los principios del nuevo modelo de Seguridad Social asentado en el art. 41 del texto constitucional, y, de otro, para dar respuesta a las exigencias continuas de adaptación a las nuevas realidades sociales y económicas. Importa recordar que en la década de los ochenta del pasado siglo se van a producir cambios normativos que afectaron a aspectos esenciales del sistema de protección social: nuevo modelo de gestión institucional (RDL 36/1978); nuevo diseño respecto de la inspección y recaudación de la Seguridad Social (Ley 40/1980 y RDL 10/1981); reformas en la pensión de jubilación (RDL 13/1981); modificación de la estructura y acción protectora de la Seguridad Social (Ley 26/1985); innovaciones en el campo de la asistencia sanitaria y establecimiento del Sistema Nacional de Salud (Ley 14/1986); modificación de la estructura financiera de la Seguridad Social (Ley 37/1988), o la introducción en el sistema de la Seguridad Social de las 
prestaciones de nivel no contributivo (Ley 26/1990), además de las modificaciones normativas que, en relación con la Seguridad Social, se fueron incorporando a través de las Leyes de Presupuestos Generales del Estado de los sucesivos ejercicios económicos (Panizo, 2016, 121).

Con la intención de dotar al ordenamiento de la Seguridad Social de una mayor sistematización e incrementar la seguridad jurídica, la Ley 26/1990 ordenó al gobierno (disposición final primera) elaborar un nuevo texto refundido de la LGSS que regularizase, aclarase y armonizase la propia ley con el texto refundido de 1974 y las disposiciones con rango de ley publicadas con posterioridad a este último, salvo las materias correspondientes al ámbito de la protección del desempleo y al sanitario, que quedaban excluidas de la refundición. Sin embargo, la disposición final segunda de Ley 22/1992, de 30 de julio, sobre medidas urgentes sobre fomento del empleo y protección por desempleo, ampliando el inicial plazo de refundición — dos años- por un año más, incluyó en la refundición la materia de desempleo, y la disposición final decimocuarta de la Ley 22/1993, de 29 de diciembre, sobre medidas fiscales, de reforma del régimen jurídico de la función pública y de la protección por desempleo, amplió el catálogo de disposiciones a refundir y armonizar, extendiendo el plazo para ello hasta el 31 de diciembre de 1994. Cuando todavía restaban seis meses para la conclusión de este plazo vio la luz el Real Decreto Legislativo 1/1994, de 20 de junio, aprobatorio del segundo texto refundido de la LGSS, si bien el TRLGSS/1974 continuó en vigor en lo relativo a la ordenación de las prestaciones sanitarias (arts. 98 a 125), conformando una especie de «islote» separado del resto de la regulación común en materia de seguridad social, que aún conserva vigencia.

Si la producción normativa en materia de Seguridad Social fue importante antes de la entrada en vigor del TRLGSS/1994, la misma se intensificó a partir de ese momento por la necesidad de adaptar el ordenamiento de la Seguridad Social a los compromisos adquiridos en los diferentes acuerdos sociales y políticos derivados del Pacto de Toledo de 1995, en orden a progresar en la simplificación estructural del sistema, pero, sobre todo, para hacer frente a los retos que imponía la crisis económica a fin de preservar la sostenibilidad del sistema en un contexto de creciente envejecimiento y mayor gasto público en pensiones. Mayor producción legislativa que iba acompañada de importantes defectos de técnica jurídica, como la decisión de ubicar en disposiciones legales diferenciadas - como las Leyes de Presupuestos y hasta 2004 en las leyes de «acompañamiento»- muchas de las reformas introducidas, sin integrarlas formalmente en 
el cuerpo del TRLGSS/1994, así como el uso '«desbocado» de las disposiciones adicionales (bien en el propio texto refundido o en leyes diferenciadas) en las que se regulaban aspectos sustantivos del sistema de Seguridad Social, en vez de incorporar esa regulación en la parte articulada del mismo, todo lo cual no hizo sino contribuir a la creación de un marco de gran incertidumbre y auténtica falta de seguridad jurídica, dificultando la interpretación y aplicación de la legislación de Seguridad Social (Panizo, 122, y Ramos, 2). Fruto de este precipitado de reformas incrustadas en el TR de la LGSS son, sin ir más lejos, sus sesenta y seis disposiciones adicionales, versadas sobre muy diversas cuestiones, que, sumadas a sus veintidós disposiciones transitorias y ocho disposiciones finales, lograron desestabilizar la estructura interna y romper la armonía de esta norma que constituye el eje del ordenamiento de la Seguridad Social.

Para intentar paliar esta situación, la disposición final vigésima quinta de la Ley 27/2011, de 1 de agosto, sobre actualización, adecuación y modernización del sistema de la Seguridad Social, volvió a facultar al gobierno para llevar a cabo una nueva refundición de la LGSS en un plazo de dos años (hasta el día 2 de agosto de 2013), a fin de integrar debidamente regularizados, aclarados y armonizados los textos legales vigentes en materia de Seguridad Social, pero el gobierno no hizo uso de esa autorización, agotándose el plazo establecido para ello.

El informe elaborado por la Comisión para la Reforma de las Administraciones Públicas (CORA) presentado al Consejo de Ministros el 21 de junio de 2013 consideraba necesaria la aprobación de una ley ordinaria que habilitara al Gobierno para elaborar textos refundidos en relación con aquellas leyes que, habiendo sido modificadas en numerosas ocasiones, destacaran por su relevancia en un área de actividad específica.

Asimismo, la Ley 19/2013, de 9 de diciembre, sobre transparencia, acceso a la información pública y buen gobierno, contiene un proyecto de revisión, simplificación y una consolidación normativa de los ordenamientos jurídicos de todas las Administraciones Públicas, para lo que habrían de efectuarse los correspondientes estudios, derogar las normas que hubieran quedado obsoletas y determinar, en su caso, la necesidad de introducir modificaciones, novedades o proponer la elaboración de un texto refundido, de conformidad con las previsiones constitucionales y legales sobre competencia y procedimiento a seguir, según el rango de las normas que quedasen afectadas.

Como parte de este proceso de revisión y simplificación normativa, la Ley 20/2014, de 29 de octubre, por la que se delega en el Gobierno la 
potestad de dictar diversos textos refundidos, haciendo uso de la técnica de delegación legislativa prevista en el art. 82 y siguientes de la Constitución, dándole para ello un plazo de doce meses a contar desde su entrada en vigor (plazo que finalizaba el 31 de octubre de 2015), autorizó en su art. uno.c) la elaboración, entre otros, de un nuevo texto refundido que recogiese debidamente aclaradas y armonizadas, además del entonces vigente texto refundido, toda una panoplia de disposiciones legales reguladoras de diferentes aspectos de Seguridad Social; labor refundidora que ha cristalizado en el TRLGSS/2015.

\section{LA DELEGACIÓN LEGISLATIVA: CONTENIDO, ALCANCE Y OBJETIVOS DE LA REFUNDICIÓN}

El ya citado art. 82 de la Constitución prevé, en su apartado 1, que las Cortes Generales pueden delegar en el gobierno la potestad de dictar normas con rango de ley sobre materias determinadas, siempre que éstas no estén afectadas por una reserva de ley orgánica; delegación que puede llevarse a cabo mediante una ley de bases, cuando lo que se pretende es la elaboración de un texto articulado, o por una ley ordinaria, si se trata de refundir varios textos legales en uno solo. Y dentro de estas última modalidad — la refundidora - el apartado 5 del mismo precepto constitucional establece dos alternativas por las que puede optar el legislador delegante: la delegación se puede limitar a la mera formulación de un texto único, o bien puede comprender también la facultad de «regularizar, aclarar y armonizar» los textos legales que vayan a ser objeto de la correspondiente refundición. Mientras que la primera opción únicamente habilita al gobierno para agrupar disposiciones legales, la segunda, sin incluir la capacidad innovadora - porque los textos refundidos no pueden innovar nada-, comporta un plus en la habilitación que va más allá de la mera transcripción sistemática de las normas vigentes con el único fin de facilitar el manejo de las mismas, pues se trata de lograr la integración de dichas disposiciones en un texto único, regularizado, aclarado y armónico, lo que a veces requiere dar una redacción que integre requisitos o condiciones que figuran en disposiciones dispersas, pero sin que ello signifique, como ha precisado el Consejo de Estado, que puedan ser acogidas en el texto refundido auténticas innovaciones que supongan un desarrollo de los preceptos que han de refundirse o que deban inspirar la refundición. 
De las dos alternativas posibles respecto de la delegación refundidora, la Ley 20/2014 ha optado por una delegación amplia, de modo que el mandato al Gobierno no se limita a la formulación de un texto único (opción que habría sido perfectamente inútil considerando la multiplicidad y falta de homogeneidad de los instrumentos legales a refundir), sino que, además, se le atribuye la facultad (y la obligación) de regularizar, aclarar y armonizar las disposiciones que han de constituir el objeto de la refundición, a fin de conseguir un texto normativo que sea entendible, sistemático y coherente, considerando que la labor refundidora no es una tarea puramente mecánica, sino que precisa a veces de ajustes para corregir errores o rectificar términos, pudiendo llegar incluso a reflejar normas adicionales y complementarias a las que son objeto de refundición, siempre que sean necesarias para colmar lagunas, precisar su sentido o dotar al nuevo texto de coherencia y sistemática (STC 13/1992, de 6 febrero, FJ 16. ${ }^{\circ}$ ).

En cuanto a la disposiciones que deben ser integradas en el nuevo texto refundido, debidamente regularizadas, aclaradas y armonizadas, son el TRLGSS/1994 — con los cambios incorporados al mismo desde su entrada en vigor- $-\mathrm{y}$ el resto de disposiciones legales enunciadas en el art. uno.c) de la Ley 20/2014, empezando por los arts. 30 y 31 de la Ley 42/1994, de 30 de diciembre, de Medidas Fiscales, Administrativas y del Orden Social, hasta el capítulo I, las disposiciones adicionales primera, segunda, tercera y cuarta, y la disposición final quinta de la Ley 23/2013, de 23 de diciembre, reguladora del factor de sostenibilidad y del índice de revalorización del sistema de pensiones de la Seguridad Social. No se refunden otros preceptos cuya entrada en vigor se ha convertido en especialmente incómoda y se viene aplazando reiteradamente (disposiciones referidas a la ampliación de la duración del permiso de paternidad, mejora en la cuantía de la pensión de viudedad en determinadas circunstancias, trabajo autónomo a tiempo parcial...), cuando podrían haberse incluido sus textos sujetándolos a una fecha de entrada en vigor pospuesta por la disposición final única (Cabero, 2016, 8).

Como refleja la Memoria del análisis de impacto normativo que acompañó al Proyecto de Real Decreto Legislativo, éste responde a la conveniencia de reordenar, aclarar y armonizar el contenido de esas normas, incluyendo en un solo texto las disposiciones legales que, relacionadas con la Seguridad Social, se fueron promulgando desde la entrada en vigor del anterior TRLGSS/1994 a través de normas complementarias y hasta el mismo momento en que se aprueba el nuevo texto refundido, logrando que éste se convierta realmente en el cuerpo legal de referencia de la Seguridad Social, 
incorporando a su contenido buena parte de las previsiones contenidas en las disposiciones adicionales del texto refundido del año 1994 o que se encontraban dispersas en otras normas legales distintas del texto refundido.

El Pleno del Consejo Económico y Social, en sesión celebrada el 23 de septiembre de 2015, hizo una valoración positiva del Proyecto de Real Decreto Legislativo remitido por el Ministerio de Empleo y Seguridad Social para emisión de dictamen sobre el mismo, pero, además de formular varias observaciones particulares, consideraba necesario profundizar en el esfuerzo de simplicidad y claridad en la sistemática y redacción de la LGSS, de modo que resulte más comprensible para sus destinatarios. Unos días más tarde (el 15 de octubre de 2015) fue el Consejo de Estado el que adoptó su dictamen sobre el proyecto de nuevo TRLGSS y, sin perjuicio de formularse diversas observaciones particulares, concluye que con él «se facilita el manejo de una legislación de especial importancia para la vida de los ciudadanos y para el trabajo de múltiples operadores jurídicos, a todos los cuales se dota de un texto de buena factura jurídica y adecuada sistemática».

\section{ESTRUCTURA Y NOVEDADES QUE PRESENTA EL NUEVO TEXTO REFUNDIDO DE LA LEY GENERAL DE SEGURIDAD SOCIAL}

Ha de diferenciarse el contenido del Real Decreto Legislativo que aprueba el TRLGSS/2015 del contenido de este último, que se inserta al final del primero.

El Real Decreto Legislativo 8/2015 consta de:

a) Un artículo único por el que se aprueba el nuevo TRLGSS.

b) Una disposición adicional donde se establece que las referencias efectuadas en otras normas a las disposiciones que han sido objeto de refundición en el nuevo TRLGSS han de entenderse realizadas a este último texto.

c) Una disposición derogatoria que deroga de forma expresa las disposiciones que se han incorporado al nuevo TRLGSS.

d) Una disposición final única que regula la entrada en vigor del nuevo TRLGSS, para la que se establece una vacatio legis hasta el 2 de enero de 2016, lo que se justifica en el hecho de haber integrado en aquel determinadas previsiones en materia de Seguridad Social contenidas en la Ley 48/2015, de 29 de octubre, de Presupuestos Generales del Estado para 
2016, que entró en vigor un día antes, el 1 de enero de 2016. No obstante, hay dos aspectos que han quedado sometidos a diversos momentos de entrada en vigor: por un lado, el complemento por maternidad por aportación demográfica a la Seguridad Social, regulado en el art. 60 del nuevo texto refundido (procedente del anterior art. 50 bis TRLGSS/1994 introducido por la Ley 48/2015), el cual será de aplicación a las pensiones contributivas de jubilación, viudedad e incapacidad permanente que se causen a partir de 1 de enero de 2016. Y, por otro lado, el factor de sostenibilidad regulado en el art. 211 del nuevo texto refundido, el cual, conforme a las previsiones del apartado 2 de la disposición final quinta de la Ley 23/2013, reguladora del factor de sostenibilidad y del índice de revalorización del sistema de pensiones de la Seguridad Social, únicamente se aplicará a las pensiones de jubilación del sistema de la Seguridad Social que se causen a partir del 1 de enero de 2019.

Por su parte, el TRLGSS/2015 consta de 373 artículos (frente a los 234 del TRLGSS/1994) agrupados en seis títulos (frente a los tres del TRLGSS/1994), subdivididos en los correspondientes capítulos, secciones y subsecciones; veintiséis disposiciones adicionales (sesenta y seis en el TRLGSS/1994), veintinueve disposiciones transitorias (por las veintidós del TRLGSS/1994) y ocho disposiciones finales (una más que en el TRLGSS/1994). A los tres títulos con que contaba el texto refundido anterior, que mantiene con las adiciones y adaptaciones pertinentes, se añaden otros tres que regulan el RETA, la prestación por cese de actividad y las prestaciones no contributivas.

De esta forma, la estructura del nuevo TRLGSS es la siguiente:

- Título I, «Normas generales del sistema de la Seguridad Social».

— Título II, «Régimen General de la Seguridad Social».

- Título III, «Protección del desempleo».

- Título IV, «Régimen Especial de Trabajadores por Cuenta Propia o Autónomos».

- Título V, «Prestación por cese de actividad».

- Título VI, «Prestaciones no contributivas».

- Disposiciones adicionales.

- Disposiciones transitorias.

- Disposiciones finales.

El resultado final es un corpus normativo más complejo y denso que el texto refundido de 1994, pero que gana en calidad técnica, sistemática 
y seguridad jurídica. La nueva norma lleva al articulado el contenido de la mayor parte de las disposiciones adicionales del TRLGSS/1994, así como otras disposiciones complementarias recogidas en leyes diferenciadas sobre temáticas muy variadas (Ley 30/1995, Ley 18/2007, Ley 27/2011, RDL 13/2010...). Lo anterior y la incorporación de la regulación relativa al RETA y a los sistemas especiales de trabajadores agrarios por cuenta ajena y el de empleados de hogar determinan que estemos ante un texto significativamente más amplio que su predecesor, la numeración de cuyos preceptos no se corresponde con la del texto refundido de 1994. Como consecuencia del encargo de regularizar, aclarar y armonizar las leyes cuya refundición se autoriza, el nuevo TRLGSS experimenta una profunda transformación de su estructura en relación con el texto refundido anterior y, por ello, también en los contenidos de buena parte de sus preceptos (Cabero, 12). Pese a todo, la técnica legislativa seguida no ha sido perfecta, como se puede apreciar en la amplitud de la «Corrección de errores» del Real Decreto Legislativo 8/2015, publicada en el BOE de 11 de febrero de 2016, que se aprovecha para subsanar alguna omisión significativa como la relativa al programa de renta activa de inserción.

El título I continúa refiriéndose a las «Normas generales del sistema de la Seguridad Social», con algunas particularidades entre las que cabe destacar: se introduce una nueva sección en el capítulo II («Campo de aplicación y estructura del sistema de la Seguridad Social») relativa a las disposiciones aplicables a determinados colectivos: familiares (art. 12), trabajadores con discapacidad (art. 13) y socios trabajadores y socios de trabajo de cooperativas (art. 14); se precisa que el deber de comprobación de los empresarios que contraten o subcontraten con otros la realización de obras o servicios correspondientes a la propia actividad de aquéllos o que se presten de forma continuada en sus centros de trabajo se circunscribe a los trabajadores que el contratista o subcontratista ocupe en dichos centros «durante el periodo de ejecución de la contrata o subcontrata» (art. 16.5); se prevé la posibilidad de inclusión de los deportistas de alto nivel (art. 7.4); se recoge la regulación del complemento por maternidad de las pensiones que fue establecido por la Ley 48/2015 (art. 60); se reproduce casi en su totalidad la regulación de las Mutuas colaboradoras con la Seguridad Social contenida en la Ley 35/2014; por lo que se refiere al régimen económico, se integra la Ley 28/2003, de 29 de septiembre, reguladora del Fondo de Reserva de la Seguridad Social, y se incluye un nuevo capítulo VIII sobre procedimientos y notificaciones que responde a la generalización del uso de los medios electrónicos. 
En lo que se refiere al Título II («Régimen General de la Seguridad Social»), y más en concreto a la extensión del campo de aplicación de dicho régimen, destacan las siguientes particularidades en el nuevo art. 136:

- El apartado 2.a) incluye a los trabajadores incluidos en el sistema especial para empleados de hogar y en el sistema especial para trabajadores por cuenta ajena agrarios.

- El apartado 2.b) se refiere a las «sociedades de capital» en lugar de «sociedades mercantiles capitalistas», adaptando la terminología a la Ley de Sociedades de Capital aprobada por el Real Decreto Legislativo 1/2010, de 2 julio.

- En los apartados 2.d) y 2.e) se incluyen como trabajadores por cuenta ajena y/o como asimilados a los socios trabajadores de las sociedades laborales, según circunstancias.

- En el apartado 2.g) se integra el apartado 1 de la disposición adicional 29. a del TRLGSS/1994 (trabajadores dedicados a las operaciones de manipulación, empaquetado, envasado y comercialización del plátano).

- En los apartados 2.k) y 2.l) se incluye al personal al servicio de las Administraciones Públicas y de las entidades y organismos vinculados o dependientes de ellas, incluido, en su caso, el periodo de prácticas (salvo que estén incluidos en el Régimen de Clases Pasivas del Estado o en otro régimen en virtud de una ley especial).

- En el apartado 2.m) se incluye al personal funcionario y otro personal de nuevo ingreso, del que se ocupa la disposición adicional $3 .{ }^{a}$ TRLGSS/2015.

- En el nuevo apartado 2.q) se mantiene la habilitación legal para la inclusión en el régimen general de cualesquiera otras personas que, por razón de su actividad, sean objeto de asimilación a trabajadores por cuenta ajena mediante real decreto, a propuesta del Ministerio de Empleo y Seguridad Social.

Respecto a las exclusiones del campo de aplicación del Régimen General (art. 137), se añaden los trabajos realizados por los profesores universitarios eméritos de conformidad con lo previsto en el apartado 2 de la disposición adicional 22. ${ }^{a}$ de la Ley Orgánica 6/2001, de 21 de diciembre, de Universidades, así como por el personal licenciado sanitario emérito nombrado al amparo de la disposición adicional 4. ${ }^{a}$ de la Ley 55/2003, de 16 de diciembre, del Estatuto Marco del personal estatutario de los servicios de salud (integra, por tanto, en parte, la DA 43. . TRLGSS/94). 
En el ámbito de la acción protectora sobresalen las siguientes especificidades:

- Se tienen en cuenta las modificaciones introducidas por la Ley 26/2015, de 28 de julio, sobre modificación del sistema de protección a la infancia y a la adolescencia, realizando las correspondientes adaptaciones terminológicas en las prestaciones de maternidad, paternidad y cuidado de menores aquejados de grave enfermedad.

- Se recupera la eventual valoración de la situación de incapacidad permanente y su graduación mediante una lista de enfermedades, que se reenvía a desarrollo reglamentario, aplicándose entretanto la normativa anterior (a cuyo efecto la disposición transitoria 26. ${ }^{a}$ del TRLGSS/2015 contiene una reducción alternativa del art. 194 del mismo, que coincide con el art. 137 del TRLGSS/1994, en la redacción anterior a la Ley 24/1997, señalando su vigencia hasta que entren en vigor las disposiciones reglamentarias oportunas).

- En el capítulo relativo a la jubilación en su modalidad contributiva se integran las modificaciones llevadas a cabo por la Ley 23/2013, reguladora del factor de sostenibilidad (art. 211), y por el RDL 5/2013, sobre promoción del envejecimiento activo (art. 214).

- Se sustituye la referencia a «sesenta y cinco o más años» por la de «sesenta y siete o más años» en aquellos preceptos en los que era necesario, dada la modificación de la edad de acceso a la jubilación ordinaria operada por la Ley 27/2011.

- El art. 223 del nuevo texto refundido lleva como rúbrica «Compatibilidad y extinción de las prestaciones de viudedad», extendiendo sus previsiones a la prestación temporal de viudedad, algo que no estaba previsto en el TRLGSS/1994, lo que ha sido caracterizado por los primeros comentaristas del nuevo texto refundido como «un exceso que no parece que esté amparado en armonización o aclaración alguna» (Monereo y Rodríguez, 2016, 21).

- Se incorpora al título II un capítulo nuevo, el XVII, para regular, en su sección 1. ${ }^{a}$, las particularidades aplicables a los trabajadores a tiempo parcial y, en la sección $2 .^{a}$, las de los trabajadores contratados para la formación y el aprendizaje.

- Se incorpora al título II un nuevo capítulo, el XVIII, sobre sistemas especiales para empleados de hogar (antes regulado en la DA $39{ }^{a}$ de la Ley $27 / 2011$ ) y para trabajadores por cuenta ajena agrarios (antes regulado en la Ley 28/2011). 
En el título III, dedicado a la protección por desempleo, se incluye un nuevo capítulo $V$ relativo a disposiciones aplicables a determinados colectivos: trabajadores incluidos en el sistema especial para trabajadores por cuenta ajena agrarios (arts. 286 a 289), trabajadores contratados para la formación y el aprendizaje (art. 290), trabajadores del Régimen Especial de los Trabajadores del Mar (art. 291) y militares profesionales de tropa y marinería (art. 292). En la determinación del objeto de la protección se especifica que no sólo se cubre la pérdida del empleo o la reducción de jornada, sino también la suspensión del contrato de trabajo (art. 262). Se sustituye la referencia al «subsidio para mayores de cincuenta y dos años» por la «subsidio para mayores de cincuenta y cinco años» y se elimina definitivamente la referencia al subsidio para mayores de cuarenta y cinco años por haber desaparecido éste, todo ello como consecuencia de las modificaciones que en su día introdujo el Real Decreto-ley 20/2012, de 13 de julio, de medidas para garantizar la estabilidad presupuestaria y de fomento de la competitividad.

En el título IV aparece la primera novedad sistemática del nuevo TRLGSS: la incorporación al mismo de la regulación legal del RETA (arts. 305-326), teniendo en cuenta, básicamente, el conjunto de disposiciones adicionales que, relacionadas con la cobertura de los trabajadores independientes, se recogían en el TRLGSS/1994, así como los contenidos de Seguridad Social del Estatuto del Trabajo Autónomo aprobado por Ley 20/2007, de 11 de julio (que siguen formalmente en vigor). El Decreto 2530/1970, de 20 de agosto, que contiene el régimen jurídico del RETA, seguirá aplicándose en lo que no se oponga a estas previsiones legales. Repárese en que la lista de inclusiones expresas en el RETA que refleja el art. 305 del TRLGSS/2015 sólo comprende los colectivos incorporados por ley, pero hay otros encuadrados en el RETA por vía reglamentaria.

Asimismo, se incorpora la protección por cese de actividad (título V), cuya regulación quedaba extramuros del TRLGSS/1994; se deroga, en consecuencia, la Ley 32/2010, de 5 de agosto, que vino a establecer dicha prestación, a excepción de lo dispuesto en sus disposiciones adicionales décima y undécima.

Las prestaciones no contributivas (familiares, invalidez, jubilación) se regulan ahora en un título propio, el título VI (arts. 351-372), dado que están desvinculadas de la pertenencia a cualquier régimen del sistema de la Seguridad Social, por lo que carecía de sentido que se incluyeran entre las prestaciones del Régimen General, como hacía el anterior texto refundido; de este modo se dota de mayor coherencia a esta vertiente prestacional del sistema. 
Por último, y dentro de las disposiciones adicionales, cabe destacar el contenido de la adicional primera, referida a la aplicación de las normas del TRLGSS/2015 a los regímenes especiales, partiendo del contenido de la disposición adicional octava del TRLGSS/1994, si bien desaparecen las referencias al RETA, al haberse incorporado la regulación del mismo dentro del título IV de aquél, por lo que las remisiones se circunscriben a los Regímenes Especiales de la Minería del Carbón y de los Trabajadores del Mar.

\section{VALORACIÓN Y PROSPECTIVA}

El texto de la Ley General de la Seguridad Social, aunque con lagunas significativas y difícilmente explicables (como la relativa a la protección social de los trabajadores del mar, regulada extramuros de aquél por la Ley 47/2015), ha salido robustecido tras su última refundición, ganando en calidad técnica, sistemática y seguridad jurídica. Ciertamente que su mayor extensión y los cambios operados en su estructura interna exigirán un periodo de adaptación por parte de los operadores jurídicos hasta que se familiaricen con el nuevo texto, pero el resultado final merece ser valorado positivamente al compilarse en uno mismo cuerpo legal normas jurídicas dispersas y de no fácil localización. Pero no concluye aquí el interminable peregrinaje normativo en materia de Seguridad Social al que venimos asistiendo desde la promulgación de la Ley de Seguridad Social de 1966. En los próximos tiempos, y al igual que sucedió a los dos textos refundidos precedentes, es más que probable que el TRLGSS/2015 encajará nuevas modificaciones para adaptarse a las cambiantes circunstancias sociales y económicas, sin ir más lejos, para preservar la sostenibilidad del sistema de Seguridad Social que es objeto de su regulación.

\section{BIBLIOGRAFÍA CITADA}

Cabero Morán, E., «Los nuevos Textos Refundidos de la Ley del Estatuto de los Trabajadores, de la Ley de Empleo y de la Ley General de la Seguridad Social (y II)», Trabajo y Derecho: nueva revista de actualidad y relaciones laborales, núm. 13 (2016), pp. 1-16.

Desdentado Bonete, A., «La nostalgia del sistema: reflexiones sobre el Derecho de la Seguridad Social en una época de crisis. A propósito del "Código de la Protección Social”», Relaciones Laborales (1996-I), pp. 473-486. 
Martín Valverde, A., «La estructura del ordenamiento de la Seguridad Social», en AAVV, Pensiones sociales. Problemas y alternativas, IX Congreso Nacional de Derecho del Trabajo y de la Seguridad Social, vol. I, Madrid, MTAS, 1999, pp. 359-373.

Monereo Pérez, J. L., y Rodríguez Iniesta, G., «El tercer texto refundido de la Ley General de Seguridad Social: significación técnico-instrumental y de política del Derecho Social», Revista de Derecho de la Seguridad Social, núm. 7 (2016), pp. 11-25.

Montoya Melgar, A., «Cincuenta años de Seguridad Social en España», Nueva Revista Española de Derecho del Trabajo, núm. 185 (2016), pp. 15-23.

Panizo Robles, J. A., «Una norma de Seguridad Social largo tiempo esperada: el nuevo Texto Refundido de la Ley General de la Seguridad Social», Trabajo y Seguridad Social. Comentarios y Casos Prácticos, núm. 394 (2016), pp. 117-140.

Ramos Quintana, M. I., «La Ley General de la Seguridad Social y su nuevo texto refundido», Trabajo y Derecho, núm. 15 (2016), pp. 1-5. 